\title{
Transformational Leadership and Systemic Thinking in an Adaptive Complex System in Management of an Organization
}

\author{
Abdolhossein Ayoubi* \\ M.Sc. Student of Medical Informatics Technology, Biomedical Engineering Department, Amirkabir University of Technology, \\ Tehran, Iran, 7th Andisheh St, Moallem Ave, Jahrom, Fars, Iran, Postal Code: 7417766486 \\ Email: ayoubi.hossein@gmail.com \\ Leila Khosravi \\ Department of Management, Islamic Azad University, Shiraz Branch, Fars, Iran \\ Majid Razeghi Jahromi
}

DBA in Strategic Management, Iran University of Industries and Mines, Tehran, Iran

\section{Doi:10.5901/mjss.2015.v6n1p245}

\section{Abstract}

With respect to the changing environment of organizations, it seems important to pay heed to the transformational leadership style. The effective and transformational leadership requires the application of systemic thinking and adaptive complex system to obtain the desired purposes. As there are inadequate number of studies done on this issue in Iran, this article examines systematic thinking, its steps, model, needed skills, transformational leadership style and the challenges of this special leadership style in a descriptive manner by the use of significant references and studies done abroad. In practice, this study can offer some suggestions to be done, and can help organizations' leaders to develop systematic thinking skills in order to be applied in practical approaches of adaptive complex system.

Keywords: Organization, Transformational Leadership, Systematic Thinking, Adaptive Complex System.

\section{Introduction}

The concept of systemic thinking refers to a set of tools (casual loop diagram, repository diagram, material -flow and data diagram and simulation models) which helps analyzer to analyze dynamic behavior of a problem. Also, systematic thinking enables individuals to shape a new point of view on events; a point of view of in which general affairs, their components and the reciprocal relationships between those components are considered more accurately (Richmond, 2013). Systemic thinking works based on its special regulations. There is a set which consists of seven cognitive approaches; skillful researchers make use of these cognitive approaches in presenting and analyzing problems. Any of these seven skills are complementary, and they are used in different steps of systematic thinking. If these seven systematic thinking skills which are the basics of systemic thinking strategy be studied in a four-stage process in an interactive way, they would be perceived more comprehensively. Each of these skills plays an important role in one or some of these stages. First, the problem or issue of applying systematic thinking is considered. Then, a theory or model is presented to clarify the reason why the problem has occurred. Then, the model or theory is investigated through simulation. If this model creates such a problem, then it needs a logic and proper theory. If it is not so, the model requires to be modified. When this modified model has established a proper theory, new findings can be presented to others so that changes may occur (Mathews \& Jones, 2008). This model is presented in FIG1. 


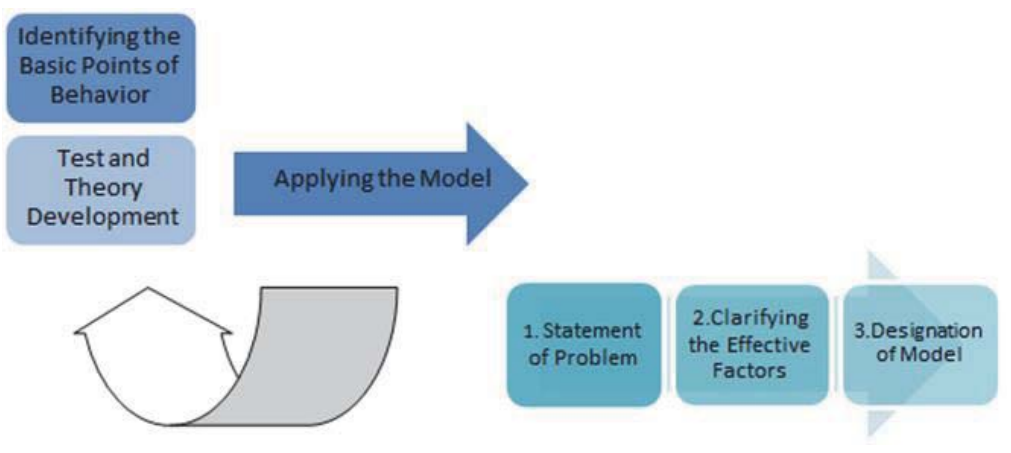

Fig. 1. The Model of Stages of Systematic Thinking ; Mathews and Jones; 2008.

Whenever the systemic thinking is used in analyzing a problem, some of the skills seem to be more salient in the stages of this approach. Based on Richmond, systemic thinkers are masters of these seven skills separately and independently. These skills are described in FIG. 2. as follows:

- First stage: identification of problem or issue and selecting boundaries of the model or theory (by the use of dynamic thinking, setting the structure as the cause, holistic thinking)

- Second stage: Creation of the model( by the use of operational thinking, closed loop thinking, quantitative thinking)

- Third stage: testing and investigating on the model(by academic thinking)

The first skill, i.e. dynamic thinking, causes the mentioned problem to be described as a phenomenon; this phenomenon is not an "event" occurring just once. The next skills, setting the structure as the cause and holistic thinking, are significant; since they help determining which aspects of reality should be included in the theory, and which details should be used in the explanations. The fourth to sixth skills, i.e. operational thinking, closed loop, and quantitative thinking can be used in presenting a theory in the format of a model which can be tested accurately. The last skill, academic thinking, is used in testing the theory. Any of these skills has a unique role in the process of systemic thinking.

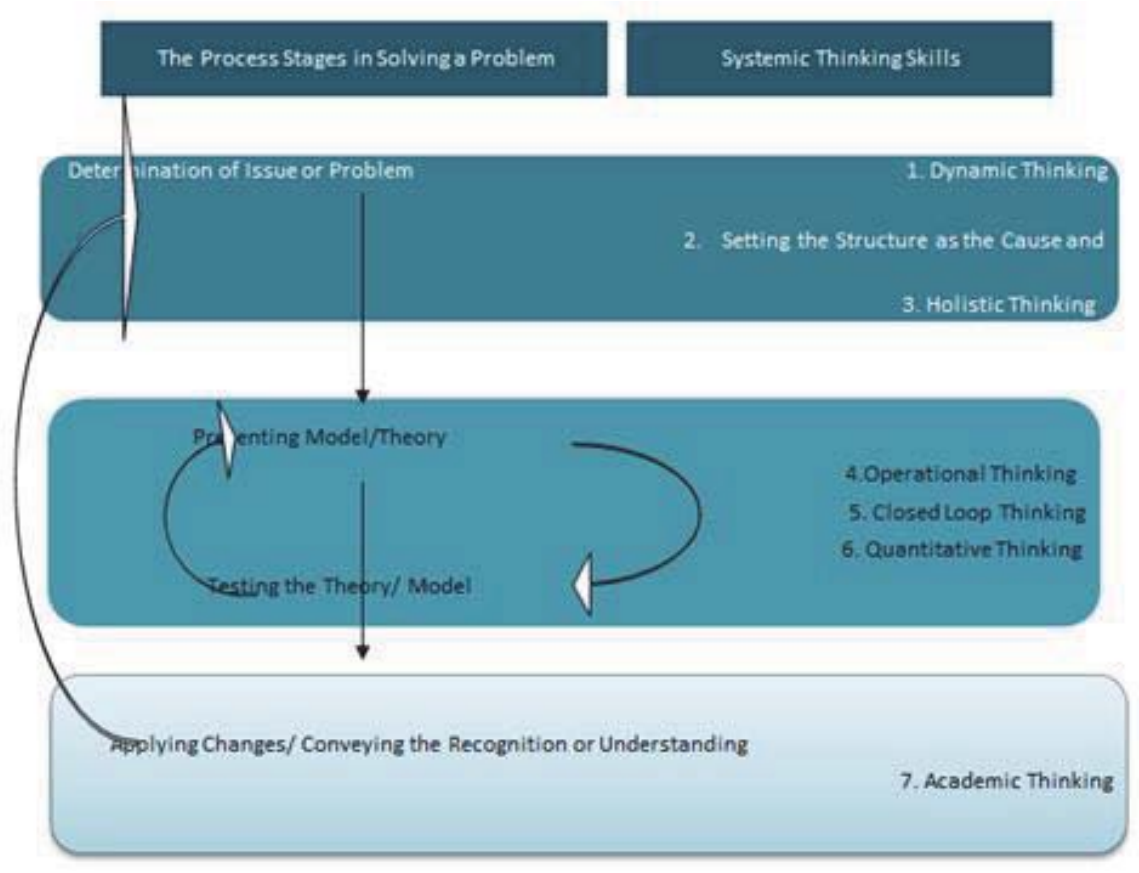

Fig. 2. The Stages and Skills of Systematic Thinking

Systematic thinking seems to be essential for establishing a learning organization, and making use of transformational 
leadership style with the tendency to learn and innovate in the organization. The role of leadership in a learning organization is to praise creativity and innovation by the use of systematic thinking (Leontes, 2013). This article intends to investigate the positive organizational results based on systematic thinking by analyzing characteristics of an impressive leader, and by preparing a learning environment to reinforce the behavioral manner.

An impressive leader of a learning organization needs to encourage the entire management group and the whole learning process; in order to do so making use of innovation and creativity to be adapted to the current changes should be increased. Nowadays that the global business environment is under the reign of stable atmosphere, the leader should move toward new ideas, continue real attempts so that innovation be used, and should provide the necessary support for the team members so that flexibility shows its faces. They key to obtain the desired results is that each person's role which is part of his productivity and is undoubtedly effective over the entire organization's productivity and effectiveness be considered through clarification of common goals. Therefore, each person's effective leadership style in the organization should be at the center of attention, and this leadership style should perform as an evolutionary or a serving style. In other words, at this exact moment the learning organization needs a transformational leader who is able to present a goal and a bright perspective according to organizational values while the benefits of employees and the stockholders are maintained (Daft, 2010).

Another key behavior of an effective and transformational leader of a learning organization is that he should improve his patience against employees' mistakes, both in inner and outer, on the other hand in stable and changing environments. Of course, this point should come into consideration that employees require a clear knowledge to be able to make a proper decision in the organization. When the acquired knowledge is considered as the employees' individual profession by the leadership of the organization, any error or mistake is assumed as an opportunity. Accordingly, a suitable leadership style may be adaptive to encouraging employees' situation; to motivate them in participating in a learning process and in practicing bravely at the time when an error has occurred. McNeil and Giescke ${ }^{1}(2004)$ define organizational leadership as a means of propagating learning, making benefit of experiences and a way to increase risktaking among employees. Daft (2010) indicates that appreciative enquiry model performs as a fine method for communicating with individuals or the whole organization in order to create new ideas by concentrating on the learning process based on the previous achievements.

\section{Applying Leadership Strategies to Assure Positive Results}

The traditional leadership strategies dictated the act of thinking or learning to the entire organization as if these processes are recipes, and needed to be followed (Senge, 2006). It should be mentioned that the global working environment is rapidly changing. The only person who can perform changes over the traditional strategies seems to be the individual employee or the manager. A lot of authors such as Singh (2013) demonstrated evolution from leadership model of administrative management to collegiate leadership model of emancipation (COLME). This model performs as a solid structure in developing leadership to gain the desired results by following leadership potential, making benefit of employees' talents, propagating responsibility and collaboration in responsiveness. As a result, in a learning organization in which systemic thinking is applied in all dimensions, any individual who is under training can be considered as a potential in the correct decision- making process; thus, the proper leadership strategy is guaranteed.

The other strategy suggests the act of developing the culture of patience and tolerance. Freely stating new ideas, i.e. comprehensive and systematic thinking culture, should be established in the organization. Garvin et al. believe that all rankings in an organization may not show interest in learning and sharing new ideas which are intrinsically in opposition with organizational culture and values. According to Singh (2013), in addition to theoretical validity which is officially accepted from a leadership model in which colleagues can freely collaborate with the learning organization, practical validity is also justified because of its compatibility with the definition of identity and expectations derived from leaders' roles. This process which is considered with the determination of the key benefits and the needs of the interest groups is contained intentionally in the organizational learning programs which are parts of the whole organizational strategic programming. Through this procedure, the hidden knowledge may conquer the written and recorded knowledge of the organization, thus its importance is revealed (Singh, 2013).

Furthermore, the well-designed methods which are based on the common values and decision-making behaviors in the construction procedure of the entire organization are similar to a simultaneous harmony observed in orchestra music which produces no disharmony or harsh sound. In general, creating a learning environment, utilizing the experiences and increasing the risk-taking abilities are considered as the key strategies in using systematic thinking in

1. Giescke, J. \& McNeil, B. 
the leading approaches (Giescke \& McNeil, 2004). In accordance with this strategy, organizational open structure may inspire employees while it pays attention to the real interests beyond the expectations. Thus, higher amount of dependency to the organization increases both self confidence and autonomy among employees, at the same time (Leppanen, 2013).

\section{Special Leadership Behaviors which Prevent the Normal Learning Process}

In his studies, Singh (2013) observed that traditional leaders utilize their administrative authority as their own right. Effective leaders tend to share their authority with their employees also to broaden the organizational rankings (Kouzes \& Posner, 1997). They may focus on the results after passing the known procedures of making employees capable while little monitoring is needed. There is no more absolute control or monitoring; in addition, preventing the changes is no more possible. The new problems and challenges can be resolved by the systemic thinking and the novel leadership style in a faster way and without any controlled sequences, indeed decision making will be processed more fluently.

As Golman (2012) indicates traditional leaders are considered as poisons to organization; they enfeeble employees' spirit and causes a sharp decrease in efficiency, also negative words and emotions get widespread while the spirit has faced a diminishing state. Lots of indicators show that the employees need to utilize their own knowledge; furthermore, they have to be more active in organizational affairs while inflexible management prohibits the learning processes and systemic thinking. In addition, leader may discover the real employees' potential by testing their individual learning style. Therefore, determining the learning materials may hurt the employees' learning abilities (Kolb et al. 1999).

Cultures of fear and the ideas being rejected cause employees to lose motivation in sharing new ideas with the leader or the management unit. Generally, if the blamed employees be compared with other teams, any tendency to collaborate and to apply new experiences will be lost. Totally, according to Northouse (2010), in contrary to cooperative and exchange management, transformational management approach in a learning organization is a process in which the communication between the leader and the followers promotes the motivation and the ethics. This special type of leadership was called stewardship by Senge (1990). The main role is played by a steward leader. The purposes of organization are clearly defined. Moreover, the leader as a steward assists the individuals in a learning organization to follow their own values and aims through which organization's aim will also be flourished.

\section{Ethical Decision-Making on Constructing the Process and the Learning Organization}

Generally, ethics may be defined as a set of principles, regulations, standards, judgments, common emotions and values related to public interests. According to Freadrich and Ferrell (2004), ethical manner includes principles and standards which lead the behaviors. Artistic style needs to be inspiring to others in the process of management in a learning organization (Senge, 2006). This style needs honesty, respect, serving others, justice and culture-building practices. Beyond social regulations, organizational working environment arranges the rules and regulations as right or wrong to the individuals who work in the organization.

Based on Meen and Keough's studies (1992), Singh declared cognitive process of reasoning as an organizational culture in any state of values. This cognitive process of learning is applied with a set of explicit knowledge. The leader is allowed to change the final decision of individual employees which undoubtedly influences on the organization's functions (Leontes, 2013). As Johnson (2012) believes ethics refers to judgment as a criterion which evaluates human behavior as right or wrong, and it can be mentioned that the best style of leadership is to greatly emphasize on the needs, values and ethics based on systematic thinking.

The goal of leadership on the basis of systematic thinking in an organization is to increase employees' creativity. So, the focus will be on providing assistance to followers, i.e. employees in order to achieve higher standards of ethical qualifications and responsibilities. According to Daft (2014), the effects of the decisions on one loop are just indicated with systemic thinking by increasing the leadership capabilities beyond the learning capabilities of arranged skills, planning or monitoring others. Also, these effects include some items which are deeper and more accurate than features and styles of leadership. It means utilizing all abilities and intellectual, emotional and psychological understanding. Any decision at any level of organization can bring unexpected results to the entire organization. It means systematic thinking teaches the probable effects of any decision to leaders and employees of that organization, not just to a special part; indeed it is conveyed that they need to think to wider aspects. Individuals and teams should be taught simultaneously since both of them are exposed to dangers at the same time.

It is worthy of noting that making benefit of a more developed loop in decision making process or using an open feedback of the loop may provide an opportunity to prevent non-compensating consequences for the whole organization 
(Haldeman, 2011). As a result, it is considered as a part of leadership behavior to support the learning environment. Such environments are useful for simple managers and employees to apply learning processes more fluently and in a more efficient way. Garvin et al. (2008) indicate systematic thinking as a primary element in the learning process since each individual in the organization plays a key role in its shaping. Creativity, innovation, tendency to maintain the learning process and sharing the explicit and the implicit knowledge, trusting to the leader and ethical behavior seem to be significant and effective to any organization; and this issue is practical from systemic thinking point of view (Leontes, 2013).

Studies indicate that the best way to equip the effective and transformational leaders who are in charge with inspecting and solving the global complexities of organization is applying systematic thinking. Any link between systematic thinking as a cognitive pattern, effectiveness of leadership behavior in different states in its simple and complex forms, augments the ability to tolerate ambiguities and diversities (Leppanen, 2013). Systematic thinking considers individual differences in cognitive pattern as a set of integrated elements which guarantee the implicit tendency in understanding a phenomenon. These elements establish dynamic patterns in a whole complex system. In the world, when a complex and competitive organization is to be survived, and also when it is to learn some lessons from another organization which exists in higher levels of systemic thinking, the decisions and responsibilities will be done in a more perfect way in the first organization.

According to studies, transformational and awakening leadership styles are utilized by the use of systematic thinking model as a cognitive pattern which originates from the best understanding of adaptive complex systems, and when these styles got fixed, they enable the leaders to move toward adaptive complex systems also to better tolerate the ambiguous systems. Analysis of leadership styles that are related to systematic thinking seems to be important to understand the special features which leaders are equipped with in the best way.

Indeed, it is proved that there's a meaningful relationship between systems of thinking as a cognitive pattern, effectiveness of leadership behavior in different states, simple and complicated, and effective transformational leadership. Awake and transformational leadership style may apply one cognitive pattern of systematic thinking to achieve the best understanding of adaptive complex systems, and when it is proved, it may allow the leaders to easily keep on with adaptive complex systems; therefore, they can tolerate the probable ambiguities and risks better (Leppanen, 2013).

When the theories of adaptive complex systems of systematic thinking went into consideration, four leadership features are suggested (Leppanen, 2013):

(1) Effective and transformational leaders can better understand the complex systems as a whole;

(2) Effective and transformational leaders may face the continuous changes by the employees' innovation and creativity in a better way;

(3) Effective and transformational leaders may recognize the significance of relations, diversity and creativity in a better way, and also they can make benefit of them as a learning opportunity;

(4) Effective and transformational leaders are capable of a better tolerance of ambiguities and confronting with probable risks.

In fact, these features are accompanied with sharing the style of effective, transformational and awake leadership. Since both of them are considered as relation-oriented; they try to provide the organizations' needs through encouraging and supporting the lower ranking followers toward upper rankings.

'Leadership' and 'transformational leadership' were termed for the first time by James Burns (1978). This leadership style indicates that leaders welcome the significant positive changes in individuals and teams by inspiring from employees' attitudes and their motivations (Warrick, 2011). These leaders enjoy the talent and the ability of leading the changes; also they can establish organizational transformations.

The leaders act based on what were mentioned above in the form of "four I" $s$ of leadership:

1. Influencing on the employees on the basis of ideals and aims while leaders act according to a pre-determined missions and clear perspectives;

2. Showing respect toward employees;

3. Being equipped to trust meanwhile both the leader and employees show higher levels of ethics;

4. And applying ethics;

All of these items are provided in this process (Leppanen, 2013).

Transformational leadership style helps leaders to be able to teach their followers how to effectively process those data related to different teams (Kearney \& Gebert, 2009). As a result, data process which is related to the work and includes developing and integrating ideas is done better (Leppanen, 2013). These benefits are more in the groups which enjoy diversity, but a leadership style which is more flexible is needed, it integrates the positive outcomes of ideas by the use of systematic thinking (Hogan \& Kaiser, 2005). It should be mentioned that these effects are in fact related to 
transformational leadership and systematic thinking in different organizational work groups.

\section{Conclusions}

This study was done to investigate the relationship between systematic thinking in effective and transformational leaders, their better leadership and their effectiveness. Since in a globalized and competitive environment, organizations need to change themselves according to the environment; this issue is put into practice based on human force, leaders and organizational managers. Meanwhile, a proper leadership style and systematic thinking reveal their significance. Systematic thinking helps suitable decisions be made in any circumstances through utilizing models, simulating and shaping a novel attitude to events while all elements and the relationships are being considered among them.

On the other hand, it is the leader of the organization who is in charge of the structure of organizational affairs. If he/she tries to see the organization or the world as a whole and as a unified set, he would gain better cognition. Also by being equipped with effective and transformational leadership, he/she would succeed in complicated organizational arranging. In fact, the leaders are those who play a significant role in being honest and in tolerating ambiguities in such systems, while they understand the important role of followers in making the open organization flexible through learning. The organization is considered as a whole and as a learning unit which seeks help from transformational and open leadership also from systematic thinking.

When the organization is involved in the ever changing and developing world, it can respond faster to the opportunities by taking advantage of creativity, innovation, rise in risk-taking, cooperation and collaboration; even the organization is now capable of transforming the risks to learning and developing opportunities. Actually, what was mentioned seems to be the adaptive complex system of systematic thinking. In this method, effective and transformational leaders obtain a better understating of the surrounding world and of themselves. Furthermore, they may be able to recognize and consequently solve the increasing complexities. Applying systematic thinking in the process of leadership is possible when systematic thinking skills are established. In order to establish these skills and applying them in the organization, some suggestions are as follows. These suggestions create opportunities to influence on the followers more and increasingly:

1. In order to establish dynamic thinking, it's better to depict diagram of attitude of organization and employees during the course of time. Events are considered as activity points in the general pattern of variables during the course of time;

2. A structure should be determined as the cause, so that reproving of employees be prevented. The way the elements caused the mistake, also the way they can show more flexibility toward problems and mistakes should be studied;

3. More attention should be paid to similarities rather than differences to achieve holistic way of thinking;

4. Identity of processes should be considered, while effective factors on these processes should be ignored so that operational thinking is established.

5. In order to establish closed-loop thinking, a check list of variables is needed; in addition the way the variables can influence on each other should be studied.

\section{References}

Burns, J. M. (1978). Leadership. New York: Harper \& Row. Cabrera, D., Colosi, L., \& Lobdell, C. (2008). Systems Thinking. Evaluation and Program Planning, 31(3), 299-310.

Daft, R. L. (2010). Organization Theory and Design (10th ed.). Mason, OH: Cengage Learning.

Haldeman, J. (2011). The learning Organization: From Dysfunction to Grace. Journal of Management \& Marketing Research, vol. 9, 1-9. Retrieved from http://ehis.ebscohost.com.library.gcu.edu:2048/eds/pdfviewer/pdfviewer?vid=6\&sid=36ae3ec5-b8d9-49c5-bd56811741d6a0f1\%40sessionmgr112\&hid=107 .

Garvin, D. et al. (2008). Is Yours a Learning Organization? Harvard Business Review, vol. 86(3), 109-116. Retrieved from http://ehis.ebscohost.com.library.gcu.edu:2048/eds/pdfviewer/pdfviewer?vid=7\&sid=91dec9f4-b8ac-4f96-a7961d267aef05fa\%40sessionmgr112\&hid=106.

Giescke, J. \& McNeil, B. (2004). Transitioning to a Learning Organization. Library Trends, vol. 53(1), 54-67. Retrieved from https://library.gcu.edu:2443/login?url=http://search.proquest.com.library.gcu.edu:2048/docview/220453827?accountid=7374.

Goldman , Alan. (2012). Corporate Cancer: Failure to Diagnose. Transforming Toxic Leaders. Retrieved from http://www. psychologytoday.com/blog/transforming-toxic-leaders/201205/corporate-cancer

Hogan, R., \& Kaiser, R. B. (2005). What We Know about Leadership. Review of General Psychology, 9(2), 169.

Johnson, C.E. (2012). Meeting the Ethical Challenges of Leadership: Casting Light or Shadow. Thousand Oaks, CA: SAGE. 
Kearney, E., \& Gebert, D. (2009). Managing Diversity and Enhancing Team Outcomes: The Promise of Transformational Leadership. Journal of Applied Psychology, 94(1), 77.

Kolb, D.A., Boyatizis, R.E., \& Mainemelis, C. (1999). Experiential Learning Theory: Previous Research and New Directions. Retrieved from http://www.leamingfromexperience.com/html/research librarv.html.

Kouzes, J. M., \& Posner, B. Z. (2003). The Leadership Practices Inventory (LPI): Participant's Workbook (Vol. 47). Pfeiffer.

Leontes, Dorzilme .( 2013). Systems Thinking: Leadership Behaviors, Ethics, and Outcomes.

Leppanen, Wendy, Stroink , . M. \&. R. Klein . ( 2013 ) . Systems Thinking as a Cognitive Component of Transformational and Awakened Leadership Styles Honours Thesis. Lakehead University .

Mathews , Leah Greden and Andrew Jonesto . (2008). Using Systems Thinking Interdisciplinary Learning Outcomes: Reflections on a Pilot Study in Land Economics.University of North Carolina Asheville, Department of Economics. Issues in integrative studies, No. 26, pp. 73-104 .

Meen, D. E. \& Keough, M. (1992). Creating the Learning Organization. McKinsey Quarterly, 58-78. Retrieved from http://ehis.ebscohost. com.library.gcu.edu:2048/eds/pdfviewer/pdfviewer?vid=7\&sid=36ae3ec5-b8d9-49c5-bd56811741d6a0f1\%40sessionmgr112\&hid=107

Senge, P. M. (2006). The fifth discipline: The Art and Practice of the Learning Organization. New York, NY: Doubleday.

Singh, P. (2013). Transforming Traditional Bureaucratic Management Practices By Employing the Collegial Leadership Model of Emancipation. International Business \& Economics Research Journal, vol. 12(8), 953-968. Retrieved from http://ehis.ebscohost.com.library.gcu.edu:2048/eds/pdfviewer/pdfviewer?vid=4\&sid=3bc36f5d-dea2-4080-b2b7b0721959e42e@sessionmgr10\&hid=7

Richmond, Barry . (2013) . An Introduction to Systems Thinking. Chapter1.www.iseesystems.com

Warrick, D. D. (2011). The Urgent Need for Skilled Transformational Leaders: Integrating Transformational Leadership and Organization Development. Journal of Leadership, Accountability, and Ethics, 8(5), 11-26. 